\title{
Ground states of anisotropic antiferromagnets with single ion and cubic anisotropy
}

\author{
T.-C. Dinh, R. Folk \\ Institut für Theoretische Physik, Johannes Kepler Universität Linz, 4040 Linz, Austria
}

Received June 15, 2009, in final form July 1, 2009

\begin{abstract}
Anisotropic antiferromagnets in an external magnetic field show a rich variety of different ground states meeting in transition lines and multicritical points. We study the dependence of the ground states of these systems in the three dimensional space on physical parameters such as exchange, single ion and cubic anisotropy. One identifies four different ground states: the paramagnetic (PM), the antiferromagnetic (AF), the spin flop (SF) and the biconical (BC) ground state. In the case of absence of a cubic anisotropy, the transition lines separating different ground states can be calculated analytically, otherwise they have to be calculated numerically. We also considered the behavior of the staggered magnetization which characterizes the different ground states. From its behavior the order of the transition from one state to the other is determined. But also the order of the transition changes along the transition lines when including the cubic anisotropy, especially at the reeentrant region where a transition from SF to BC and back to SF by increasing the external field $H$ occurs. Multicritical points are found which are assumed to be tricritical or critical endpoints. The results obtained may be relevant for other systems since the antiferromagnetic model can be mapped to a lattice gas model where the biconical ground state is interpreted as supersolid phase. Recent renormalization group calculations show that such a phase would indicate the existence of a tetracritical point.
\end{abstract}

Key words: antiferromagnetism, quantum lattice gas, ground states

PACS: $75.10 . H \mathrm{k}, 75.30 . \mathrm{Kz}, 67.80 . \mathrm{bd}$

\section{Introduction}

Anisotropic systems in an external field not coupled to the order parameters (OP) characterizing the condensed phases show interesting phase diagrams depending on the values of the anisotropy and the external field. One example is the anisotropic antiferromagnet in an external magnetic field [1]. Other ones are crystal systems undergoing a displacive phase transition under external pressure [2]. The possible variety of phases at finite temperature is also determined by the topology of the ground state phase diagram since the phases corresponding to different ground states extend to finite temperatures. However, at finite temperature new phases not present at $T=0$ may arise [3]. Due to the variety of possible ground states or phases multicritical points of different kind are possible at finite $T$. Using the magnetic language these systems show antiferromagnetic (AF), spinflop (SF), biconical $(\mathrm{BC})$ and paramagnetic $(\mathrm{PM})$ ground states and phases. The transition lines between these phases may meet in one point: (i) the bicritical point, where three phases coexist (AF, SP, and PM) and (ii) the tetracritical point, where four phases $(\mathrm{AF}, \mathrm{BC}, \mathrm{SP}$, and PM) coexist. Going beyond the mean field theory [3] the nature of the multicritical points has been studied within renormalization group theory [4] (in a one loop order calculation). These results have been questioned by higher loop calculations $[5,6]$. Recently both using simulation methods $[7,8]$ and renormalization group theory $[9,10]$ the nature of the multicritical point has been reconsidered.

It is well known that there is a correspondence between such magnetic model and the quantum lattice gas [11] which allows to transpose the results obtained for the magnetic system to systems like $\mathrm{He}^{4}$ with superfluid and supersolid phases [12]. The question of the existence of such phases is of general interest.

Symmetry considerations are essential for the existence and the nature of ground states and phases. This is also the case for the systems treated here. It has been found that interaction terms of cubic symmetry have a strong effect on the phase diagram [13]. Here we present the results at 
$T=0$ which clarifies the order of the transition lines between different ground states. Moreover, we extend the models studied so far by combining several types of anisotropy.

\section{Single ion anisotropy}

Let us first consider the well studied case of an antiferromagnet with the Hamiltonian $\mathcal{H}$

$$
\mathcal{H}=J \sum_{\langle i j\rangle}^{N_{A}, N_{B}}\left(\Delta\left(S_{i x} S_{j x}+S_{i y} S_{j y}\right)+S_{i z} S_{j z}\right)-H\left(\sum_{i}^{N_{A}} S_{i z}+\sum_{j}^{N_{B}} S_{j z}\right)+D\left(\sum_{i}^{N_{A}} S_{i z}^{2}+\sum_{j}^{N_{B}} S_{j z}^{2}\right)
$$

It describes $N_{A}$ and $N_{B}$ interacting classical spins $\vec{S}_{i}$ on two sublattices $A$ and $B$, where $N_{A}=N_{B}$. The anisotropy of the positive exchange interaction $J$ is characterised by the parameter $\Delta$ with $\Delta=0$ for a magnet where only the $z$-components of the three dimensional spins interact and $\Delta=1$ for an isotropic magnet. The spins are subjected to an external magnetic field $H$ and a single-ion anisotropy $D$.
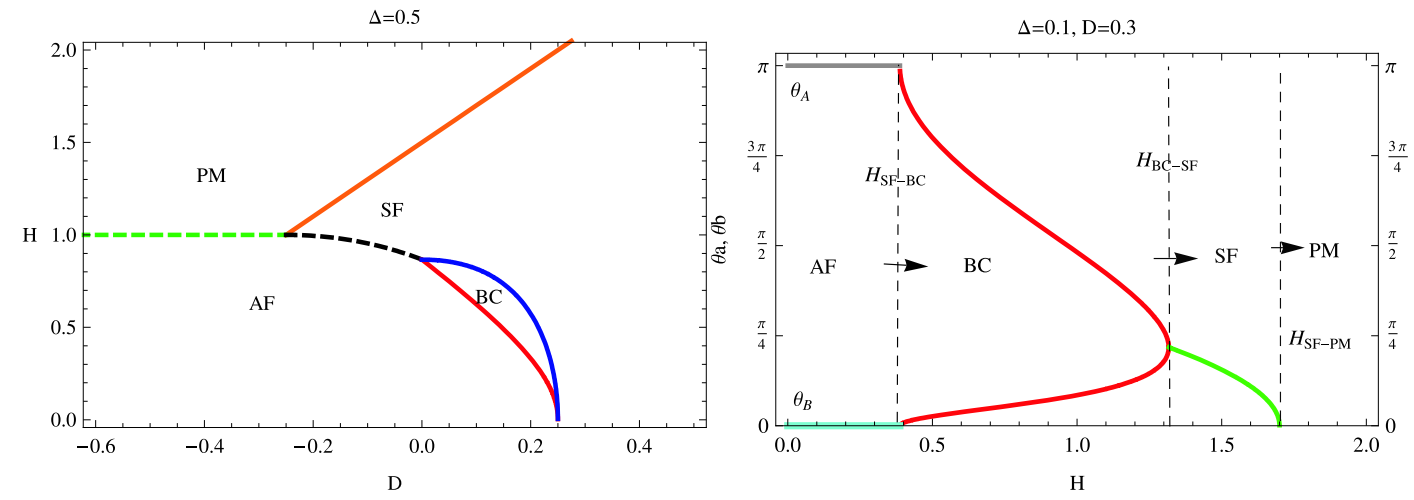

Figure 1. Left: The phase diagram for the XXZ-Model including the single-ion-anisotropy. Right: The order parameter of the four ground states configuration can be calculated analytically.

The case where beside the exchange anisotropy only a single-ion anisotropy is present can be solved analytically [14] at $T=0$. A BC ground state exists for a certain region of positive $D$ and external magnetic field $H$ for $\Delta=0$. This region becomes smaller for increasing $\Delta$ and diminishes for an isotropic antiferromagnet with $\Delta=1$. The transition lines between the AF to the PM and between the AF and the SF ground state are of the first order. The other transitions are of the second order. Figure 1 shows the whole ground state diagram with the parameters chosen so that a $\mathrm{BC}$ ground state appears. Here we divide the parameters $H, D, F$ by the number of next neighbors $(z=6$ for simple cubic system), so in order to compare with the results in $[13,15]$ one has to multiply all the three parameters in this paper with 6 . Also the OP for the complicated BC ground state can be calculated exactly which reads:

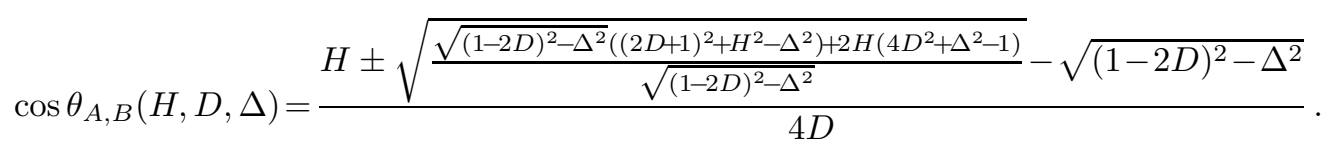

An extension to finite temperature within mean field theory has been discussed in [3]. As already explained in [11] and used in [14] a quantal lattice gas can be mapped on an anisotropic antiferromagnet with a Hamiltonian of form (1). The correspondence of different ground states is given in table 1.

In the $H-T$ ground state diagram the ground states at $T=0$ extend as phases to higher temperature. Since for high enough temperature and large enough external magnetic field the PM phase should be the stable one, all other phases are confined to finite regions in the phase diagram. 
Table 1. Correspondence between the phases of the classical magnetic system and those of the quantum lattice gas (QLG). Also shown are the order parameters, the alternating magnetization in the field direction $N_{\|}$and perpendicular to it $N_{\perp}$, and the components of the magnetization $M_{\|}$and $M_{\perp}$.

\begin{tabular}{|c|c|c|c|c|c|}
\hline Phase & $N_{\|}$ & $N_{\perp}$ & $M_{\Perp}$ & $M_{\perp}$ & QLG \\
\hline $\mathrm{AF}$ & $\neq 0$ & 0 & 0 & 0 & solid \\
$\mathrm{SF}$ & 0 & $\neq 0$ & $\neq 0$ & 0 & superfluid \\
$\mathrm{BC}$ & $\neq 0$ & $\neq 0$ & $\neq 0$ & $\neq 0$ & super solid \\
$\mathrm{PM}$ & 0 & 0 & $\neq 0$ & 0 & fluid \\
\hline
\end{tabular}

Thus it happens that the phase transition lines intersect each other in multicritical points. This might be a bicritical point when the AF, the SF and the PM phase meet, or a tetracritical point where the $\mathrm{AF}$, the $\mathrm{SF}$, the $\mathrm{BC}$ and the $\mathrm{PM}$ phase meet. It should be noted that this is even possible if the $\mathrm{BC}$ phase does not extend to $T=0[3]$.

\section{Cubic anisotropy}

Uniaxial antiferromagnets may also have cubic anisotropy. Here we consider an anisotropic antiferromagnet with a cubic anisotropy $F$ instead of the single-ion anisotropy $D$. For such a system the Hamiltonian reads

$$
\begin{aligned}
\mathcal{H}= & J \sum_{\langle i j\rangle}^{N_{A}, N_{B}}\left(\Delta\left(S_{i x} S_{j x}+S_{i y} S_{j y}\right)+S_{i z} S_{j z}\right)-H\left(\sum_{i}^{N_{A}} S_{i z}+\sum_{j}^{N_{B}} S_{j z}\right) \\
& +F\left(\sum_{i}^{N_{A}}\left(S_{i x}^{4}+S_{i y}^{4}+S_{i z}^{4}\right)+\sum_{j}^{N_{B}}\left(S_{j x}^{4}+S_{j y}^{4}+S_{j z}^{4}\right)\right)
\end{aligned}
$$
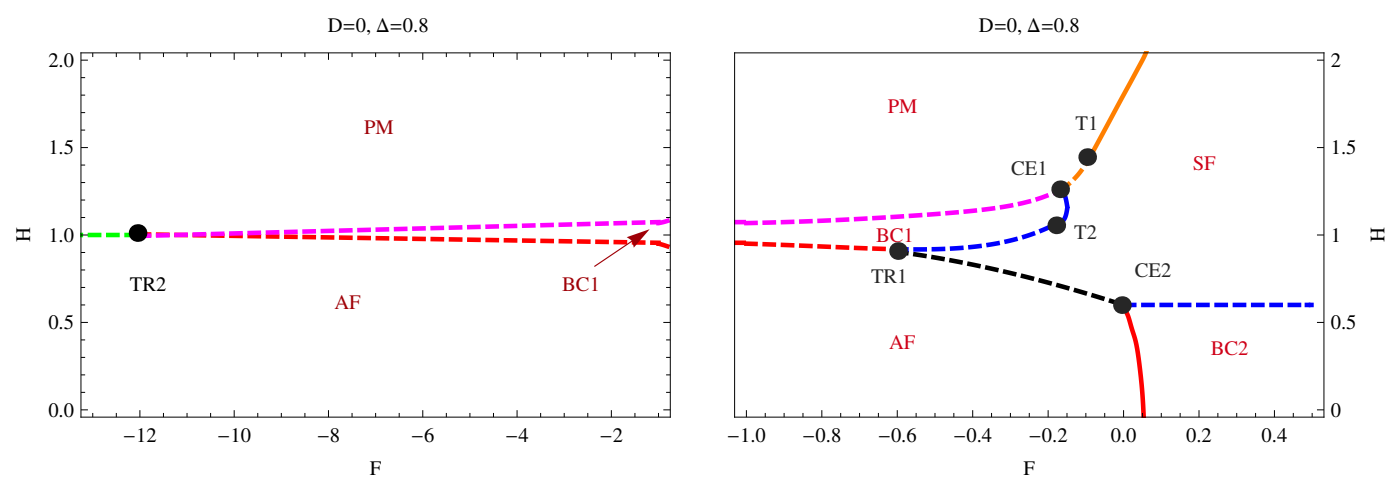

Figure 2. Ground states minimizing the energy, equation (3), in the magnetic field $H$ and cubic anisotropy $F$ plane at an exchange anisotropy $\Delta=0.8$ and with zero single ion anisotropy $D$. The ground states are denoted as follows: AF antiferromagnetic, PM paramagnetic, SF spin flop ; $\mathrm{BC} 1,2$ biconical oriented along the diagonal or the axis in the $x-y$-plane. Shown are special points where either more than two ground states meet or where the order of the transition line changes from the first (dashed line) to the second order (solid line). These are the triple points $\mathrm{TR} 1,2$, the tricritical points $\mathrm{T} 1,2$ and the critical end points $\mathrm{CE} 1,2$.

The effect of such a type of the cubic anisotropy has been studied recently by $[13,15]$. It has been shown that two types of $\mathrm{BC}$ ground states are possible (BC1 for $F<0$ and $\mathrm{BC} 2$ for $F>$ 0 ), depending whether the perpendicular component of the alternating magnetization lies in the 
diagonals or along the axes in the $x y$-plane. Moreover, it has been found that most of the transition lines between the ground states become first order. There was also found a reentrant region for the SF ground state becoming unstable for increasing the external magnetic field with respect to the BC state, which for even larger field again becomes unstable with respect to the SF phase (see figure 2). In this reentrant region the transition line has been found to be of second order.
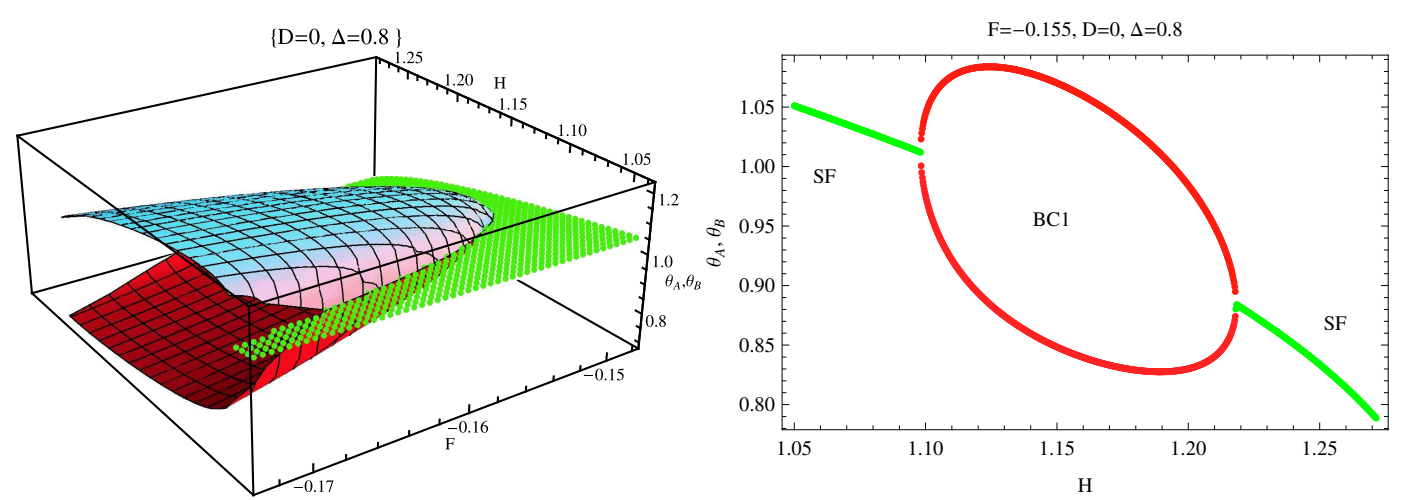

Figure 3. A $3 \mathrm{D}$ and a $2 \mathrm{D}$ diagram showing the occurrence of the reentrant region by increasing the magnetic field $H$.

We verify the occurrence of the reentrant region [13] by calculating the order parameter of the ground states which take place in the vicinity of this region (see figure 3 ). The $2 \mathrm{D}$ figure shows that the SF-BC1 occurs by increasing the applying field $H$. In case of further increasing the field, the $\mathrm{SF}$ reappears again.
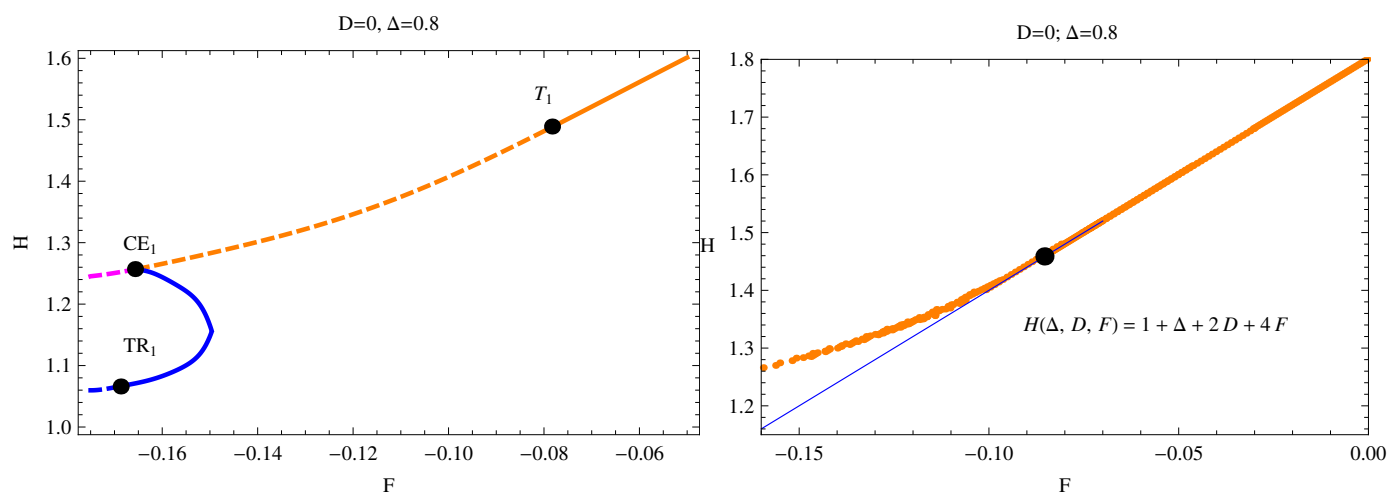

Figure 4. Left: Detail from the right part of figure 1 on a larger scale around the reentrant region. Solid lines are of the second order, dashed lines of the first order. The second order reentrant line between the biconical and spin flop ground state starting at the tricritical point TR1 ends in the critical end point CE1 on the transition line to the paramagnetic ground state. On the transition line between the paramagnetic and spin flop ground state one finds the tricritical point T1. Right: Comparison of the transition line between the SF and PM ground state calculated on the assumption of a second order transition (straight line) and the transition line calculated numerically by minimizing the energy. Indeed when the transition curve deviates from the second order line the transition becomes the first order.

In order to find out the transition points where a change in the order of the transition takes place we have to calculate the nonzero order parameter component along the border lines for the case treated in [13]. Along the transition line between the $\mathrm{BC} 1$ and the $\mathrm{PM}$ ground state the parallel staggered magnetization $N_{\|}$goes to zero at the point where the SF-PM and the BC1-SF transition lines meet (see figure 2). The behavior of the OP is compatible with a power law with 
a correction term

$$
\left|N_{\perp, \|}\right|=A\left|\frac{\left|F(H)-F^{\star}\right|}{F^{\star}}\right|^{0.5}\left(1+B\left|\frac{F(H)-F^{\star}}{F^{\star}}\right|\right),
$$

where $F^{\star}$ is the value of the cubic anisotropy at the special point, and $F(H)$ the value of the cubic anisotropy along the transition line. $A, B$ and $F^{\star}$ are fit parameters. This point is a critical end point (CE1). From this critical endpoint the second order reentrant line starts. This line ends in a point where the $\mathrm{AF}$, the $\mathrm{BC} 1$ and the $\mathrm{SF}$ ground state meet. This point turns out to be a triple point (TR1) since the second order character of the reentrant line is changed to the first order (at T2) before it reaches the triple point. The value of $N_{\|}$becomes nonzero on the reentrant line at $\mathrm{T} 2$ and this point might be a tricritical point (see figure 5).

We also find a change of the order along the transitions line between the SF and the PM ground state. At T1 the transition becomes the second order for larger values of $F(H)$. This is just at the point where the transition line begins to deviate from the analytically calculated second order transition line (see figure 5).
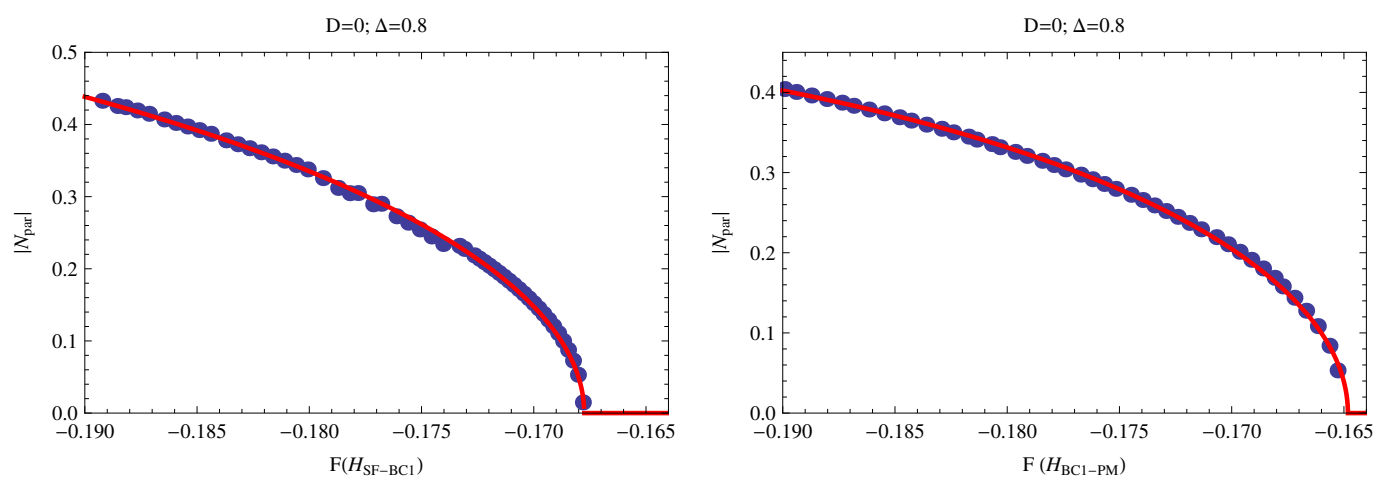

Figure 5. Left: Change of the parallel order parameter $\left|N_{\|}\right|$going along the first order transition lines between the $\mathrm{BC} 1$ and $\mathrm{PM}$ ground states. The dots are the calculated values and the solid line is a fit according to equation (4). The zero value of the parallel OP is reached at the critical end point CE1. Right: Similar the change of the perpendicular order parameter $\left|N_{\perp}\right|$ going along the first order transition lines between the SF and PM ground state. The zero value of the perpendicular OP is reached at the tricritical point $\mathrm{T} 1$.

Conclusively we summarize all the critical points in a table (see table 2)

Table 2. Summary of all the special points discovered in the ground state diagram for $D=0$ and $\Delta=0.8$. There exist tricritical points $(\mathrm{T})$, tripel points $(\mathrm{TR})$ and the critical end points $(\mathrm{CE})$.

\begin{tabular}{ll|ll}
\hline Critical Points & Coordinates $(F, H)$ & Critical Points & Coordinates $(F, H)$ \\
\hline T1 & $(-0.087747,1.49)$ & TR2 & $(-12,1)$ \\
T2 & $(-0.167755,1)$ & CE1 & $(-0.164823,1.26)$ \\
TR1 & $(-0.6,1.6)$ & CE2 & $(0,0.6)$ \\
\hline
\end{tabular}

\section{Single ion and cubic anisotropy}

Let us now consider the general case when the exchange, single-ion and cubic anisotropy are present

$$
\mathcal{H}=J \sum_{\langle i j\rangle}^{N_{A}, N_{B}}\left(\Delta\left(S_{i x} S_{j x}+S_{i y} S_{j y}\right)+S_{i z} S_{j z}\right)-H\left(\sum_{i}^{N_{A}} S_{i z}+\sum_{j}^{N_{B}} S_{j z}\right)
$$




$$
+D\left(\sum_{i}^{N_{A}} S_{i z}^{2}+\sum_{j}^{N_{B}} S_{j z}^{2}\right)+F\left(\sum_{i}^{N_{A}}\left(S_{i x}^{4}+S_{i y}^{4}+S_{i z}^{4}\right)+\sum_{j}^{N_{B}}\left(S_{j x}^{4}+S_{j y}^{4}+S_{j z}^{4}\right)\right) .
$$

Then the planar ground state regions extend to three dimensional regions and whole lines of multicritical points appear. In figure 7 we give as an example the case where the single ion anisotropy has been chosen nonzero $(\Delta=0.8)$ and a sketch of the three dimensional ground state diagram. An analytic result for the transition line between the SF ground state and the PM ground state can be calculated assuming a second order transition, which of course is just correct in certain section of the transition line (see figure 4). The relation reads

$$
H(\Delta, D, F)=2 D+4 F+\Delta+1 .
$$

Also in zero magnetic field $(H=0)$ the stable ground states as a function of the anisotropies can be calculated analytically. The transition between the $\mathrm{AF}$ and the $\mathrm{BC} 2$ ground state starts at a positive value of the cubic anisotropy $F$

$$
F(D, \Delta)=(1-2 D-\Delta) / 4
$$

The transition occurs just only in the region $D<\frac{1-\Delta}{2}$ and is continuous (see figure 6), where the OP parameter of the biconical ground state $(\mathrm{BC} 2)$ can be given.

$$
\theta_{A, B}=\arccos \left( \pm \frac{1+2 F-2 D-\Delta}{6 F}\right) .
$$

This 2nd order transition continues to be also valid when applying the magnetic field. Increasing the single ion isotropy $\left(D>\frac{1-\Delta}{2}\right)$ the Spin-Flop is preferred to the $\mathrm{BC} 2$ and the transition takes place at

$$
F(D, \Delta)=\frac{-1+2 D+\Delta}{2}
$$

If the cubic anisotropy is zero, no transition to the $\mathrm{BC} 1$ ground state takes place.
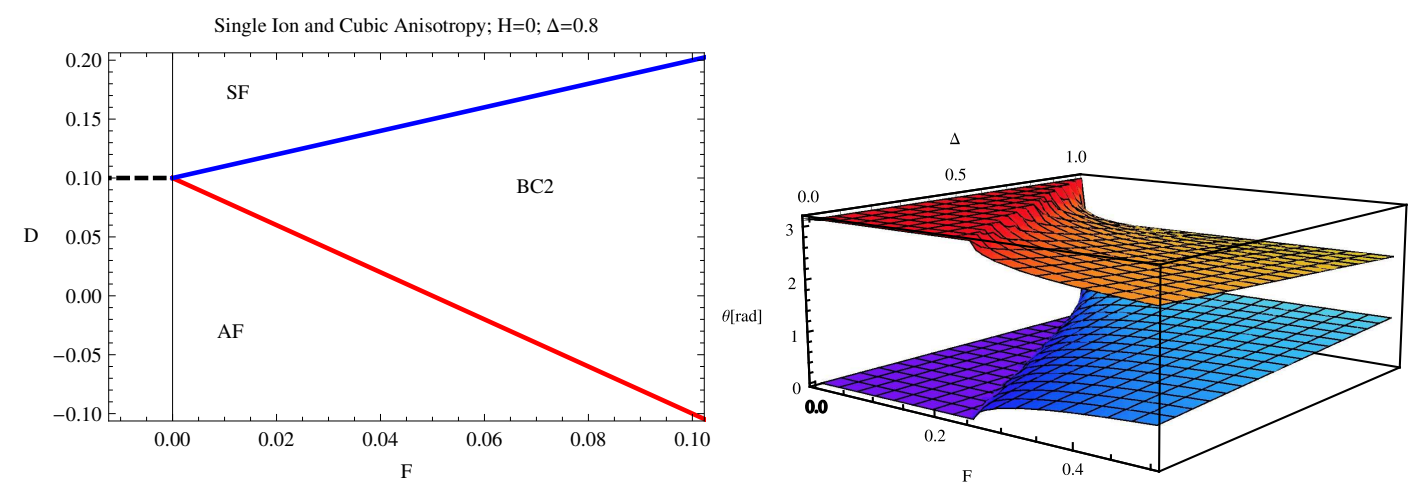

Figure 6. Left: The analytically calculated ground state diagram for $H=0$. Right: The continuous transition from AF to BC2. The two polar angles, from which the OP can be calculated, of the two different sublattices are shown.

For negative cubic anisotropy in zero magnetic field the transition from the AF to the SF state takes place at

$$
D(F, \Delta)=\frac{1}{2}(1-\Delta)
$$

and is independent of $F$. 


\section{Conclusion and outlook}

A system of an anisotropic antiferromagnet shows lots of interesting ground states and critical points. It can also be mapped into a quantum lattice system where the biconical ground state is interpreted as the supersolid state. We have shown that for the simple case where just the exchange $(\Delta)$ and the single-ion anisotropy $(D)$ are considered, one can analytically calculate all the ground states. In the case with cubic anisotropy the resulting Hamiltonian is more difficult to be solved, so that we have to calculate the ground states numerically. In this system two kinds of biconical ground states appear, the $\mathrm{BC} 1$ for $F<0$ and $\mathrm{BC} 2$ for $F>0$.

Most of the transition lines are of the first order, but in some cases the order of the transition also changes along the transition line. So, lots of interesting critical points appear ( critical endpoint, tricritical point). For example, along the transition line between $\mathrm{BC} 1$ and $\mathrm{SF}$ for $F<0$ a reentrance region can be shown where the order of the transition changes from the second to the first order by increasing the absolute value of the cubic anisotropy.

However, in the absence of the field $H$ we can derive the transition surfaces analytically, because the term with the applied field $(H)$ is the part of the Hamiltonion which breaks the symmetry and, therefore, causes this Hamiltonian unsolvable. The BC2 ground state does also exist at $H=0$ for a large value of $F$.

The topology of the ground state diagram at $T=0$ determines the possible multicritical points at finite temperatures. Without cubic anisotropy bicritical or tetracritical points are possible where two lines of the second order phase transitions with order parameters of dimension $n=1$ and $n=2$ meet. Cubic anisotropy may change this picture and multicritical point where two lines with $n=1$ and $n=3$ or $n=3$ and $n=2$ might meet (see figures 2,7 for positive values of $F$ ). Cubic anisotropy changes most of the transitions to the first order, however on the transition line separating the BC1 from the SF ground state, part of this line starting at a critical end point is of the second order type. Moreover, part of the transition line separating the spinflop state from the paramagnetic state is of the second order.
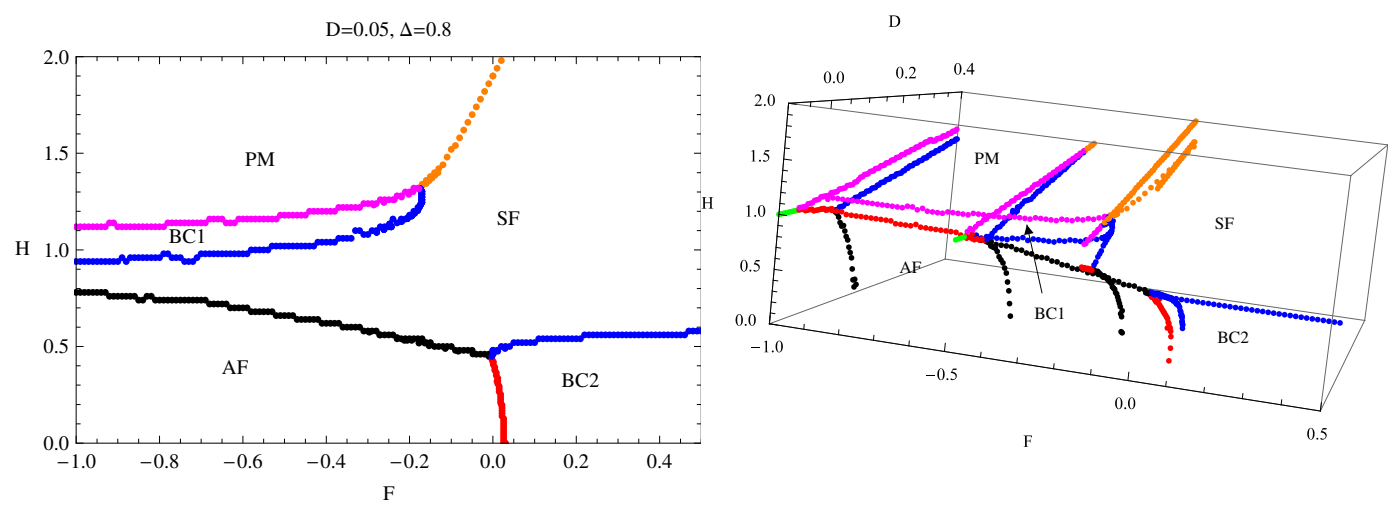

Figure 7. Left: Ground states minimizing the energy, equation (5), in the magnetic field $H$ and cubic anisotropy $F$ plane at an exchange anisotropy $\Delta=0.8$ and with single ion anisotropy $D=0.05$. Right: A 3D-phase diagram $H(F, D)$ with fix single-ion-anisotropy $\Delta=0.8$.

\section{Acknowledgements}

We thank W. Selke for valuable discussions. This work was supported by the Fonds zur Förderung der wissenschaftlichen Forschung under Project No. P19583-N20. 


\title{
References
}

1. Shapira Y. Multicritical Phenomena. Plenum, New York and London, 1983, p. 35; for a more recent review see Aharony A., J. Stat, 2003, 110, 659.

2. Bruce A.D., Aharony A., Phys. Rev. B, 1975, 11, 478.

3. Liu K.-S., Fisher M.E., J. Low Temp. Phys., 1973, 10, 655.

4. Nelson D., Kosterlitz J.M., Fisher M.E., Phys. Rev. Lett., 1974, 33, 813.

5. Prudnikov V.V., Prudnikov P.V., Fedorenko A.A., JETP Lett., 1998, 68, 950.

6. Calabrese P., Pelissetto A., Vicari E., Phys. Rev., 2003, 67, 054505.

7. Selke W., Holtschneider M., Leidl R., Wessel S., Bannasch G. Computer Simulation Studies in Condensed Matter Physics XXI, eds. D. P. Landau, S.P. Lewis, and H. B. Schüttler. Springer-Verlag, Heidelberg, Berlin, 2008.

8. Holtschneider M. Thesis RWTH Aachen, 2007.

9. Folk R., Holovatch Yu., Moser G., Phys. Rev. E, 2008, 78, 041124.

10. Folk R., Holovatch Yu., Moser G., Phys. Rev. E, 2008, 78, 041125.

11. Fisher M.E., Rep. Prog. Phys., 1967, 30, 615.

12. Peters D., McCulloch I.P., Selke W., Phys. Rev. B, 2009, 79, 132406.

13. Bannasch G., Selke W., Eur. Phys. J. B, 2009, 69, No. 3, 432 [Preprint arXiv:cond-mat/0807.1019].

14. Matsuda H., Tsuneto T., Prog. Theor. Phys., 1970, 40, 411.

15. Bannasch G. Diploma Thesis RWTH Aachen, 2008.

\section{Основні стани анізотропних антиферомагнетиків 3 одно-іонною та кубічною анізотропією}

\author{
Т.-Ц. Дінг, Р. Фольк \\ Інститут теоретичної фізики, Університет ім. Йогана Кеплера, 4040 Лінц, Австрія
}

Отримано 15 червня 2009 р., в остаточному вигляді - 1 липня 2009 р.

\begin{abstract}
Анізотропні антиферомегнетики у зовнішніх магнітних полях проявляють велике різноманіття основних станів, що межують на лініях фазових переходів і в мультикритичних точках. Тут досліджується залежність основних станів у таких тривимірних системах від параметрів обмінної взаємодії, одноіонної та кубічної анізотропій. Індентифіковано чотири різні основні стани: парамагнітний (ПМ), антиферомагнітний (АФ), з нахиленими спінами (НС) та двоконусний (ДК). У випадку відсутності кубічної анізотропії лінії фазових переходів, що розділяють різні основні стани, можуть бути розраховані аналітично; в іншому випадку їх можна розрахувати лише чисельно. Розглянуто також поведінку ступінчастої намагніченості, типову для різних основних станів. Аналізуючи ії̈, визначено рід фазового переходу з одного стану до іншого. Проте рід фазового переходу змінюється також вздовж лінії переходу при включенні кубічної анізотропії, особливо в подвійній області , де при збільшенні зовнішнього поля $H$ відбувається спершу перехід від НС до ДК, а далі - знову до НС. Знайдено мультикритичні точки, які слід думати будуть трикритичними або ж критичними кінцевими точками. Отримані результати можуть бути важливими і для інших систем, оскільки антиферомагнітну модель можна відобразити у модель граткового газу, де двоконусний основний стан інтерпретується як надплинний твердотільний. Нещодавні ренормгрупові розрахунки вказують, що така фаза мала б сигналізувати про існування чотирикритичної точки.
\end{abstract}

Ключові слова: антиферомагнетизм, квантовий гратковий газ, основні стани

PACS: $75.10 . H k, 75.30 . K z, 67.80 . b d$ 